\section{An Update on NCCN/NBGH Initiative to Develop Evidence-Based Cancer Treatment Recommendations: Year 3}

\author{
Elizabeth Danielson, MHA
}

For the past 2 years, NCCN has worked with The National Business Group on Health (NBGH) to develop An Employer's Guide to Cancer Treatment 8 Prevention (the Guide). The Guide addresses issues related to cancer across the continuum of care, including prevention and wellness; medical, pharmacy, and behavioral health benefits; employee assistance programs; and disability and family medical leave. Recommendations are supported by evidence and knowledge from a wide range of experts so that employers using the Guide can be confident that benefit dollars are invested wisely and meet the needs of employees and their dependents.

The entire set of tools that make up the Guide can now be found on NCCN. org. Of particular interest is Tool 2, which includes all benefit recommendations, the rationale for them, and detailed administrative guidance.

This project will benefit individuals with employer-sponsored benefits and treating physicians and nurses who care for individuals with cancer. The project advances understanding among employers about the importance of offering comprehensive coverage for evidence-based cancer treatment and clinical trials; access to needed care, whether in the community or at an NCI-designated cancer center; and coverage for services before cancer treatment, such as fertility treatment, when relevant.

In 2013, the third and final year of the project, work will focus on wellness, cancer risk reduction, cancer screening, and beneficiary communicationsstrategies and resources to help individuals make informed decisions and cope with their own or a loved one's cancer diagnosis. A beneficiary communication resource, The Cancer Benefits and Resource Guide, will be posted on the NCCN and NBGH websites in autumn of 2013. Once it is available, employers will be able to customize the tool with their company's name and logo and edit it to include employer-specific benefit information. When the Guide is customized, it will provide employees with information they need to know about employer-sponsored benefits and services that are likely to be useful during the continuum of cancer-related care as depicted in Figure 1 -from prevention and screening through survivorship or end of life.

This Guide will also provide links to credible, recognized sources of information and support for employees dealing with their own or a loved one's cancer diagnosis and/or treatment. By making informed decisions, patients can feel more in control and will have a better chance of good outcomes, benefitting both employees and employers.

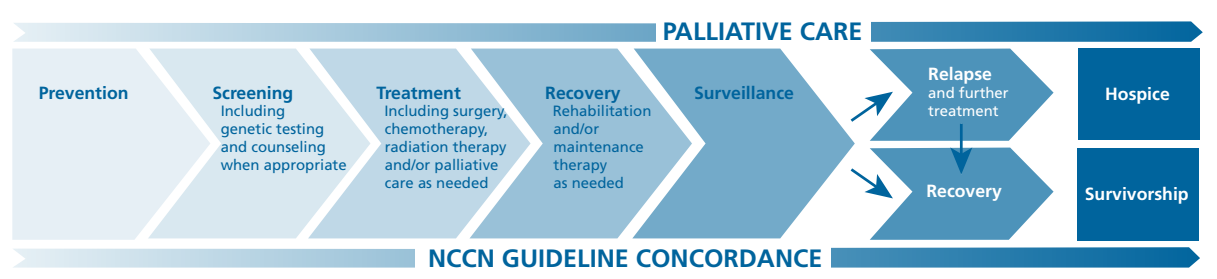

Figure 1 An example of the continuum of cancer-related care.

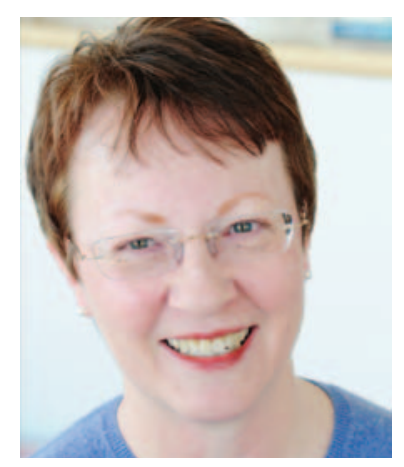

Elizabeth Danielson, MHA

Elizabeth (Liz) Danielson,

MHA, is Director of Payer \& Employer Initiatives at NCCN, where she is responsible for developing, maintaining, and expanding working relationships between NCCN and managed care organizations, employers, and other payer organizations nationwide. Her responsibilities include providing consulting services related to product development, benefit design, and implementation. She is also responsible for the development and implementation of clinical training programs for payer case managers, medical directors, and others within these organizations.

Ms. Danielson has 25 years of experience in the health care industry. Before joining NCCN in 2008, she was Director of Cancer Programs at OptumHealth, a UnitedHealth Group Company, and before that, Administrative Director of the Blood and Marrow Transplant Program at the University of Minnesota.

She earned a Bachelors' degree and a Masters of Healthcare Administration (MHA) from the University of Minnesota.

Ms. Danielson is the lead NCCN representative on the described project with the National Business Group on Health.
The ideas and viewpoints expressed in this editorial are those of the author and do not necessarily represent any policy, position, or program of NCCN. 
Danielson

About the National Business Group on Health

The National Business Group on Health (NBGH) is the nation's only non-profit organization devoted exclusively to representing large employers' perspective on national health policy issues and providing practical solutions to its members' most important health care and health benefits challenges. NBGH members are primarily Fortune 500 companies and large publicsector employers that provide health coverage for more than 40 million United States workers, retirees, and families. The NBGH fosters the development of a safe, high-quality health care delivery system and treatments based on scientific evidence of effectiveness. Members share strategies for controlling health care costs, improving patient safety and quality of care, increasing productivity, and supporting healthy lifestyles. For more information, visit www. businessgrouphealth.org.

\section{Content Overview for The Cancer Benefits and Resource Guide}

Patients need to make several important decisions fairly quickly after being diagnosed with cancer. The decisions they make will have a significant impact on their quality of care and quality of life, as well as how they handle recovery or an incurable cancer. Topics addressed include:

- Understanding the diagnosis

- Choosing a doctor

- Considerations for getting a second opinion

- Deciding on the right treatment

- Questions to ask the doctor

- Understanding the patient's health plan and other benefits

- Finding resources for support and guidance

- Planning ahead (the importance of completing an advance directive, durable power of attorney and will)

- Dealing with work-related issues (including workplace accommodation, leave options, returning to work, what to say to coworkers)

- Recovery and survivorship

The Cancer Benefits and Resource Guide will also address issues relevant for employees dealing with a loved one's cancer, including the impact of taking on a caregiver role, cancer risk reduction, recommended cancer screening, and understanding when genetic counseling is appropriate.

A companion document will focus on the needs of employers and include information to help supervisors and managers address workplace challenges. Supervisors and managers need information and resources to help them manage issues that may arise when a member of the work team is dealing with his or her own or a loved one's cancer diagnosis or other serious or chronic illness. 